\title{
Molecular behaviors in thin film lubrication-Part three: Superlubricity attained by polar and nonpolar molecules
}

\author{
Xiangyu GE ${ }^{1}$, Tobias HALMANS ${ }^{1,2}$, Jinjin $\mathbf{L I}^{1, *}$, Jianbin LUO ${ }^{1, *}$ \\ ${ }^{1}$ State Key Laboratory of Tribology, Tsinghua University, Beijing 100084, China \\ ${ }^{2}$ RWTH Aachen University, Aachen 52062, Germany \\ Received: 14 July 2018 / Revised: 25 September 2018 / Accepted: 29 October 2018 \\ (C) The author(s) 2018. This article is published with open access at Springerlink.com
}

\begin{abstract}
In thin-film lubrication (TFL), generally, the viscosity of the lubricant and its coefficient of friction $(\mathrm{CoF})$ increase. Finding a method to reduce the $\mathrm{CoF}$ in TFL is a significant challenge for tribologists. In the present work, we report a robust superlubricity attained by using polyalkylene glycols (PAGs, polar molecules) and poly- $\alpha$-olefins (PAOs, nonpolar molecules) as lubricants on steel/steel friction pairs that have been pre-treated by wearing-in with polyethylene glycol aqueous solution (PEG(aq)). A steady superlubricity state with a CoF of 0.0045 for PAG100 and 0.006 for PAO6 could be maintained for at least $1 \mathrm{~h}$. Various affecting factors, including the sliding velocity, normal load, and viscosity of the lubricants, were investigated. Element analysis proved that composite tribochemical layers were deposited on the worn region after the treatment with PEG(aq). These layers were formed by the tribochemical reactions between PEG and steel and composed of various substances including oxides, iron oxides, $\mathrm{FeOOH}$, and $\mathrm{Fe}(\mathrm{OH})_{3}$, which contributed to the superlubricity. In addition to the tribochemical layers, ordered layers and a fluid layer were formed by the PAGs and PAOs during the superlubricity periods. All the three types of layers contributed to the superlubricity, indicating that it was attained in the TFL regime. Accordingly, a mechanism was proposed for the superlubricity of the PAGs and PAOs in the TFL regime in this work. This study will increase the scientific understanding of the superlubricity in the TFL regime and reveal, in the future, the potential for designing superlubricity systems on steel surfaces for industrial applications.
\end{abstract}

Keywords: thin film lubrication; superlubricity; PEG; PAG; PAO

\section{Introduction}

Since the hydrodynamic lubrication (HDL, 1886) theory was proposed by Reynolds [1], boundary lubrication (BL, 1921) [2] and elasto-hydrodynamic lubrication (EHL, 1949) [3] have been proposed to distinguish different lubrication regimes. For a thick film, the contact surfaces are separated completely by a fluid layer, which makes the ordered layers less important and leads to a lubrication regime that shows EHL behavior [4,5]. When the thickness of a film is decreased to a few nanometers, the fluid layer and ordered layers are destroyed, only the adsorbed layers (or tribochemical layers) function between the contact surfaces, and BL [6] is established there. Clearly, a transition regime is expected between EHL and $\mathrm{BL}$, where the fluid layer, ordered layers, and adsorbed layers (or tribochemical layers) coexist and function to reduce the friction. Decades later, a new lubrication regime with this three-layer structure was proposed and named as thin-film lubrication (TFL, 1996) [7, 8]. The molecular model of TFL has been directly proven by experimental detection [9]. By controlling the alignment of nematic liquid crystal molecules in a nanogap, a nano-sandwich structure consisting of an adsorbed layer, an ordered-molecule layer, and a

* Corresponding authors: Jinjin LI, E-mail: lijinjin@mail.tsinghua.edu.cn; Jianbin LUO, E-mail: luojb@tsinghua.edu.cn 
fluid layer was demonstrated, which was a typical TFL model [9]. The TFL theory depicts the entire map of the lubrication regimes as well as provides insight into the molecular form in the confined liquid layers [8]. In the TFL regime, the thickness of the ordered layers is related to the initial viscosity and molecular size of the lubricant, polarity of the lubricant molecules, and characteristics of the solid surfaces [7]. Other factors that can change the orientation of the molecules, such as an external electrical field, can also change the film thickness and friction characteristics in TFL [10]. The latest research has extended the thickness of TFL to approximately $200 \mathrm{~nm}$ [11], and the thin-film behavior has been studied under very a high pressure [12] and at starvation conditions [13] with inlet replenishment mechanisms [14].

However, generally, the lubricant viscosity in the TFL regime can increase the coefficient of friction (CoF) owing to the confined effect. Therefore, identifying a method to reduce the CoF in TFL is still a significant challenge for tribologists. In Part One of this serial work, polar additives in nonpolar base oils were found to exhibit an enrichment effect in the Hertz contact region. The enrichment of the additive molecules could enhance the film-forming ability of a lubricant and reduce $\mathrm{CoF}$ by over $60 \%$ [15]. However, the friction is still in a traditional range. Because significant progress has been made for reducing friction, a lubrication state that provides super low friction or sliding resistance is proposed and named as superlubricity. In general, superlubricity is defined as a state in which the $\mathrm{CoF}$ is less than 0.01 owing to the measurement precision level [16]. Substantial research studies on superlubricity have been conducted [17-19]. Some are on liquid superlubricity with oil-based lubricants, such as poly$\alpha$-olefins (PAOs), applied to $\mathrm{Si}_{3} \mathrm{~N}_{4} /$ diamond-like carbon (DLC) film friction pairs [20] and on castor oil applied to Nitinol 60 alloy/steel friction pairs [21] in the BL regime. Glycerol is known to provide superlubricity to a DLC film because it can easily slide on the triboformed $\mathrm{OH}$-terminated surfaces [22]. Additionally, glycerol can achieve superlubricity between steel/steel friction pairs in environments having various humidity levels [22] or with nano-diamond additives [23]. Roomtemperature ionic liquids, which are of tremendous interest in both fundamental and applied research in the lubrication field, have been found to provide superlubricity [24, 25]. In addition, some acids such as phosphoric acid aqueous solutions $\left(\mathrm{H}_{3} \mathrm{PO}_{4}(\mathrm{aq})\right)$, have been reported to achieve superlubricity [26]. The superlubricity mechanism of $\mathrm{H}_{3} \mathrm{PO}_{4}(\mathrm{aq})$ is based on the synergy effect of the $\mathrm{H}^{+}$ions and a hydrogenated network. However, the strong acidity of this type of a lubricant limits its applications in engineering. Recently, a type of weak acid (boric acid) was found to provide superlubricity when mixed with a polyethylene glycol aqueous solution (PEG(aq)) [27]. The superlubricity of this lubricant can be attributed to the equilibrium between the production and consumption of $\mathrm{H}^{+}$ions, making the superlubricity achievable under neutral conditions.

Even though liquid superlubricity has been attained and investigated in both the BL $[6,21,28]$ and EHL regime [29], it has been barely investigated in the TFL regime. To solve this issue related to the TFL regime, in this work, a robust superlubricity state with a superlow $\mathrm{CoF}$ of 0.004 was attained in the TFL regime by the formation of tribochemical layers on the contact surfaces via a pre-wearing-in treatment. Polyhydric alcohols are known to exhibit excellent friction reduction between steel/steel friction pairs because of triboformed $\mathrm{OH}$-terminated surfaces [22, 23]. Among the polyhydric alcohols, PEG has proved to be a good lubricant [27], and thus, its aqueous solution was used in the wearing-in process. Steel and commercial oils were selected as the friction pairs and lubricants, respectively, with the objective of making advances in industrial lubrication. The robust superlubricity was investigated and analyzed in detail, and a possible model of superlubricity in the TFL regime was developed.

\section{Experimental conditions}

PEG, four types of PAGs (polar molecules), and four types of PAOs (nonpolar molecules) were used, and their chemical structures are presented in Fig. 1. PEG with $99.5 \%$ purity was purchased from Sinopharm Chemical Reagent Co., Ltd. The PAGs (purity>99\%) and PAOs (purity $>99 \%$ ) were purchased from Dow Chemical Company and Exxon Mobil Corporation, respectively. No further treatment of these lubricants was performed. PEG(aq) was composed of $33 \mathrm{wt} \%$ PEG and $67 \mathrm{wt} \%$ pure water (resistivity $>18 \mathrm{M} \Omega \cdot \mathrm{cm}$ ). 

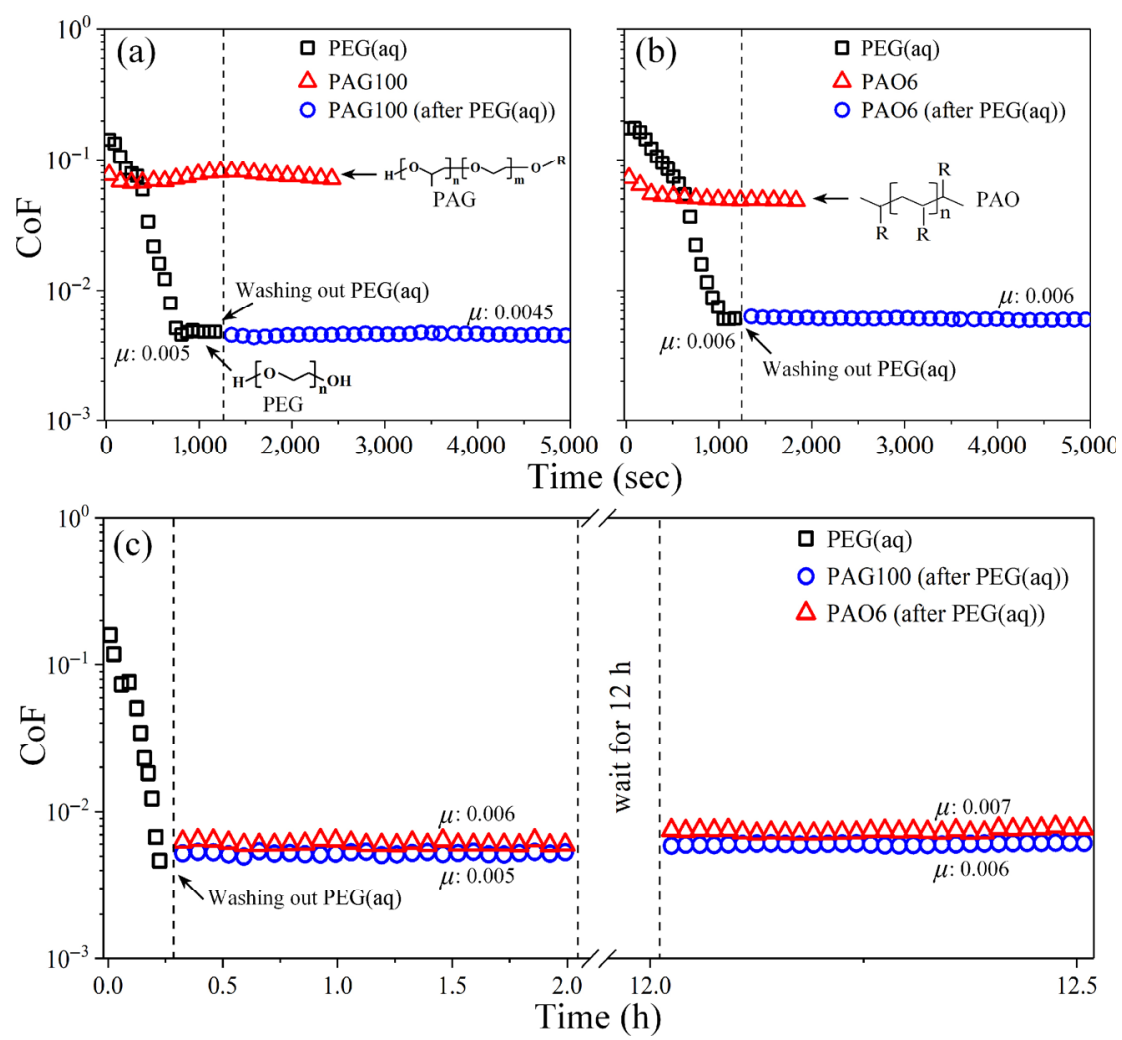

Fig. 1 (a) CoF of PEG(aq) and PAG100 on untreated steel/steel friction pairs and of PAG100 on steel/steel friction pairs treated by PEG(aq). The insets are the chemical structures of PEG and PAG. (b) CoF of PEG(aq) and PAO6 on untreated steel/steel friction pairs and of PAO6 on steel/steel friction pairs that have been treated by PEG(aq). The inset is the chemical structure of PAO. (c) Long-duration experiment of the lubricants; after the achievement of superlubricity for $2 \mathrm{~h}$, the experiment was paused for $12 \mathrm{~h}$ before restarting. The normal load and sliding velocity were $3 \mathrm{~N}$ and $0.2 \mathrm{~m} / \mathrm{s}$, respectively.

A Universal Micro-Tribotester (UMT-5, Bruker, USA) was used to perform the pure sliding friction tests using a rotating ball-on-disk model. The 20-mm-diameter upper steel ball and lower steel disk (with roughness (Ra) of approximately $10 \mathrm{~nm}$ and $15 \mathrm{~nm}$, respectively) were made of AISI52100 steel. Before conducting the experiments, the steel balls and disks were first washed in ethanol and acetone, then washed in pure water, and finally dried in an air oven. The volume of the lubricant used in each test was $50 \mu \mathrm{L}$. The normal load between the friction pairs ranged from 2 to $4 \mathrm{~N}$ (corresponding to contact pressure $370-470 \mathrm{MPa}$ ), and the sliding velocity of the steel disks varied from 0.0125 to $0.25 \mathrm{~m} / \mathrm{s}$. The levelness of the rotating platform was regulated to achieve similar CoFs in both clockwise and counterclockwise rotations, providing test results accurate to within \pm 0.001 . All the tests were conducted at $24-27^{\circ} \mathrm{C}$ and with a relative humidity of $10 \%-20 \%$, and each sample was tested thrice.
A standard rheometer (Physica MCR301, Anton Paar) was used to measure the viscosity of each lubricant at $25^{\circ} \mathrm{C}$. The worn scar diameter (WSD) and roughness of the worn region were probed with a three-dimensional (3D) white-light interferometry microscope (ZYGO, Nexview). The worn region topography was obtained using a scanning electron microscope (SEM, Quanta 200 FEG). Furthermore, the chemical elements presented in the worn region were probed via X-ray photoelectron spectroscopy (XPS) to explore the chemical characteristics of the tribofilm.

\section{Results and discussion}

The steel/steel friction pairs were treated by PEG(aq) in advance by the following method. First, $50 \mu \mathrm{L}$ PEG(aq) was injected in the pre-cleaned steel/steel friction pairs. Next, a normal friction test was conducted under $3 \mathrm{~N}$ and $0.2 \mathrm{~m} / \mathrm{s}$ to perform the wearing-in 
process for these friction pairs. When the CoF of PEG(aq) decreased to less than 0.01 and was maintained constantly for $3 \mathrm{~min}$, the friction test was stopped, and the steel/steel friction pairs were cleaned with pure water, yielding the steel/steel friction pairs were to be used.

The CoF of PEG(aq) during the wearing-in process is presented in Fig. 1(a). It can be seen that the CoF decreases from 0.2 to 0.005 after a wearing-in period of $800 \mathrm{~s}$ and maintains at 0.005 for $200 \mathrm{~s}$. After this wearing-in treatment by PEG(aq), the worn region was cleaned by pure water to wash out the remaining PEG(aq). Next, PAG100 and PAO6 were injected to the same wear track and their CoFs were measured again between the steel/steel friction pairs that were treated by PEG(aq). It is found from this figure that the CoFs of PAG100 and PAO6 directly decrease to approximately 0.0045 and 0.006 , respectively, by an order of 0.001 . This is referred to as superlubricity, as exhibited in Figs. 1(a) and 1(b). Therefore, superlubricity can be attained by using PAG100 and PAO6 to lubricate friction pairs that have been treated by PEG(aq) in advance. Moreover, the superlubricity states of both PAG100 and PAO6 are maintained for at least $1 \mathrm{~h}$, indicative of the robustness of the superlubricity state.

To study the differences between the friction pairs that were and were not treated by PEG(aq), the lubricity of PAG100 and PAO6 on the latter friction pairs was studied as well. The results show that neither PAG100 nor PAO6 can attain superlubricity on the untreated friction pairs. The CoFs of PAG100 and PAO6 remain at approximately 0.07 and 0.05 , respectively. These results confirm that the treatment of friction pairs by PEG(aq) plays the key role in realizing superlubricity. Moreover, another experiment was conducted to examine the life time of the superlubricity state. The procedure of the achievement of superlubricity was the same; the difference was that after the superlubricity state was maintained for $2 \mathrm{~h}$, the experiment was paused for $12 \mathrm{~h}$ with no change and then the experiment was restated. It can be seen in Fig. 1(c) that the superlubricity states for both PAG100 and PAO6 are still stable even though the experiment is paused for $12 \mathrm{~h}$ in the absence of shear and pressure, indicative of the robustness of the lubricating film in its natural state.

To study the relationship between the sliding velocity and $\mathrm{CoF}$ during the superlubricity period, the CoFs of PAG100 and PAO6 between the steel/steel friction pairs that were treated by PEG(aq) were measured at various sliding velocities, as presented in Fig. 2(a). When the sliding velocity increases from 0.0125 to $0.1 \mathrm{~m} / \mathrm{s}$, the CoFs of both PAG100 and PAO6 decrease rapidly from 0.1 to 0.01 . When the sliding velocity further increases from 0.15 to $0.25 \mathrm{~m} / \mathrm{s}$, the CoFs of both PAG100 and PAO6 decrease to less than 0.01, entering the superlubricity state. Moreover, PAO6 yields a slightly higher CoF than PAG100 for sliding velocities higher than $0.1 \mathrm{~m} / \mathrm{s}$. For PAG100 and PAO6, the minimum average $\mathrm{CoFs}$ during the superlubricity period are 0.0043 and 0.0062 , respectively, which were correspondingly obtained at $0.2 \mathrm{~m} / \mathrm{s}$ and $0.15 \mathrm{~m} / \mathrm{s}$. Both the CoFs slightly increase when the sliding velocity is higher than the velocity at which the minimum average $\mathrm{CoF}$ is obtained. Therefore, after the steel/steel friction pairs are treated by PEG(aq), both PAG100 and PAO6 can realize superlubricity when the sliding velocities are higher than $0.15 \mathrm{~m} / \mathrm{s}$. The shapes of the both curves seem to be consistent with those of the typical Stribeck curve, which indicates that the superlubricity state is between the BL and EHL regimes. To reveal the relationship between the normal load and CoF during the superlubricity period, both PAG100 and PAO6 were tested under various normal loads ranging from 2 to $4 \mathrm{~N}$ at a constant sliding velocity $(0.2 \mathrm{~m} / \mathrm{s})$, as shown in Fig. 2(b). Both the lubricants can achieve superlubricity under all the five normal loads. The minimum CoFs of PAG100 and PAO6 during their superlubricity periods are 0.004 and 0.006, which are obtained under normal loads of $2 \mathrm{~N}$ and $3 \mathrm{~N}$, respectively. Moreover, the $\mathrm{CoF}$ of PAG100 slightly increases with increasing normal load, whereas that of PAO6 remains nearly constant.

PAGs and PAOs with four different viscosities were tested and compared to study the effect of viscosity on lubricity. As exhibited in Fig. 2(c), when the used friction pairs are not treated by PEG(aq), the CoFs of PAGs decrease with increasing viscosity and all the PAGs yield average CoFs between 0.05 and 0.1 . The same phenomenon applies to the PAOs, whose CoFs decrease from 0.1 to 0.07 with increasing viscosity. When the used steel/steel friction pairs are treated by PEG(aq) in advance, there is a significant CoF reduction for all of the PAGs and PAOs, as presented 

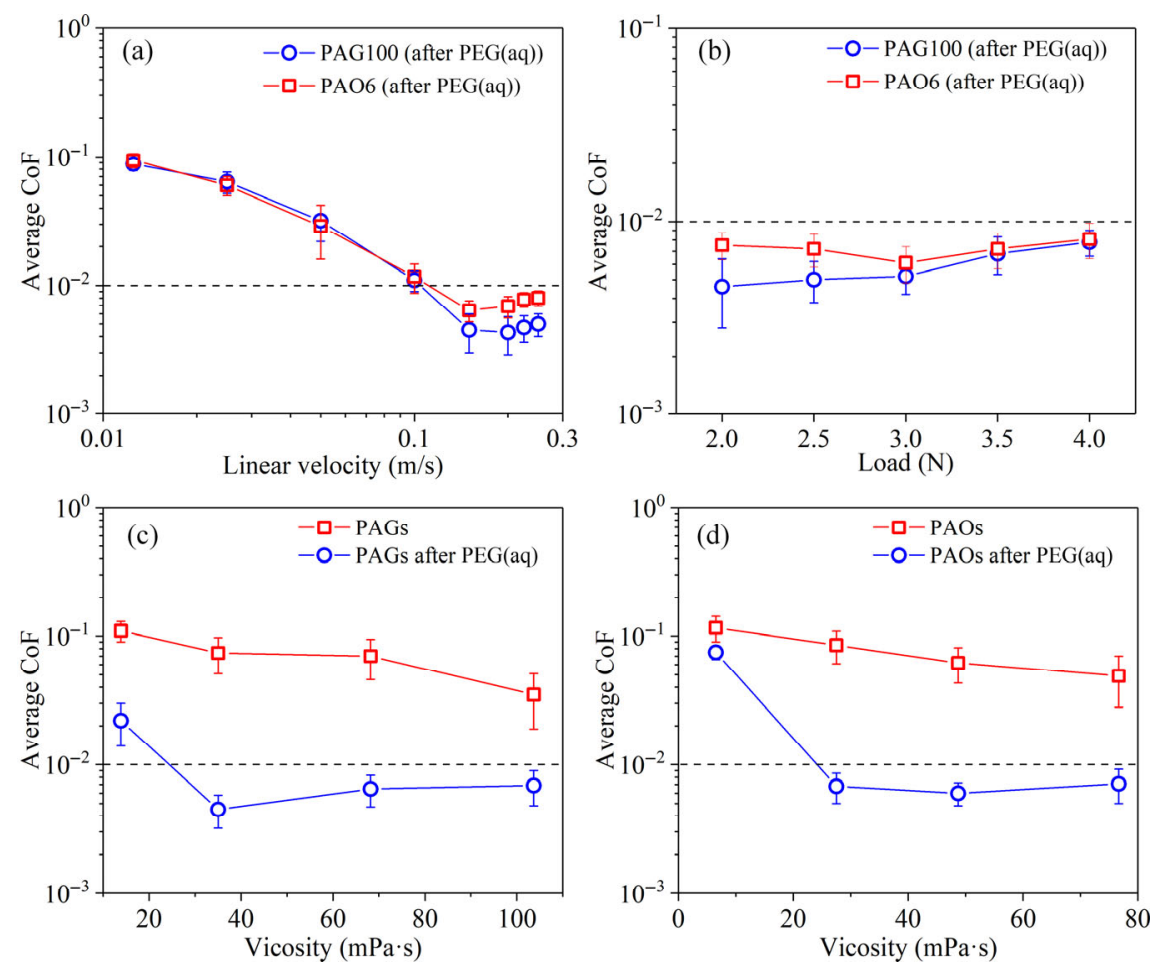

Fig. 2 Average CoFs during the superlubricity periods of (a) PAG100 (and PAO6) with respect to sliding velocity, where the steel/steel friction pairs have been treated by PEG(aq) in advance and the normal load was $3 \mathrm{~N}$; (b) PAG100 (and PAO6) with respect to normal load, where the steel/steel friction pairs have been treated by PEG(aq) in advance and the sliding velocity was $0.2 \mathrm{~m} / \mathrm{s}$; (c) the PAGs with respect to viscosity; and (d) the PAOs with respect to viscosity. For (c) and (d), both steel/steel friction pairs that have been treated by PEG(aq) and ones that have not been treated were used, and the normal load and sliding velocity were $3 \mathrm{~N}$ and $0.2 \mathrm{~m} / \mathrm{s}$, respectively.

in Figs. 2(c) and 2(d). When the viscosity of the lubricant is less than $20 \mathrm{mPa} \cdot \mathrm{s}$, then owing to the low viscosity, both PAG and PAO yield relatively high CoFs ( $>0.01)$. This is because according to HamrockDowson (H-D) theory [30], it is difficult for them to generate hydrodynamic films at such low sliding velocities and under high normal loads. When the viscosity of both PAGs and PAOs is higher than $30 \mathrm{mPa} \cdot \mathrm{s}$, CoFs less than 0.01 (the benchmark for superlubricity) can be obtained between the steel/steel friction pairs that are treated by PEG(aq). The minimum CoFs during the superlubricity periods of PAG and PAO are 0.0045 and 0.006 , which are obtained at $35 \mathrm{mPa} \cdot \mathrm{s}$ and $49 \mathrm{mPa} \cdot \mathrm{s}$, respectively. These results indicate that when the PAGs and PAOs are used to lubricate the steel/steel friction pairs that are pre-treated by PEG(aq), their CoFs can be affected by the fluid effects. Therefore, a fluid layer is required between each steel/steel friction pair, which can influence the lubricities of the PAGs and PAOs.

To determine how the PEG(aq), PAGs, and PAOs affected the CoFs during the superlubricity period, the worn regions lubricated with PEG(aq) as well as those(which were treated by PEG(aq) in advance) lubricated with PAG100 and PAO6 were observed using a 3D white-light interferometry microscope and SEM, as presented in Fig. 3. $R a$ is $42 \mathrm{~nm}$ for the worn region on the steel ball lubricated with PEG(aq), and it remains nearly unchanged after lubrication with PAG100 $(R a \approx 46 \mathrm{~nm})$ and PAO $(R a \approx 39 \mathrm{~nm})$. The WSD of the steel ball lubricated with PEG(aq) was approximately $490 \mu \mathrm{m}$ (corresponding to a contact pressure of $15 \mathrm{MPa}$ ), as exhibited in Fig. 3(a), and it remains nearly the same after lubrication with PAG100 and PAO6, as exhibited in Figs. 3(b) and 3(c), respectively. These results indicate that once superlubricity is attained, no wear can occur during the superlubricity period. Enlarged images of the worn regions of the steel balls are presented in Figs. 3(d)-3(f). It can be seen that after treatment by PEG(aq), the worn region (Fig. 3(d)) is rough with a few clear grooves.

When using PAG100 to lubricate the friction pairs 


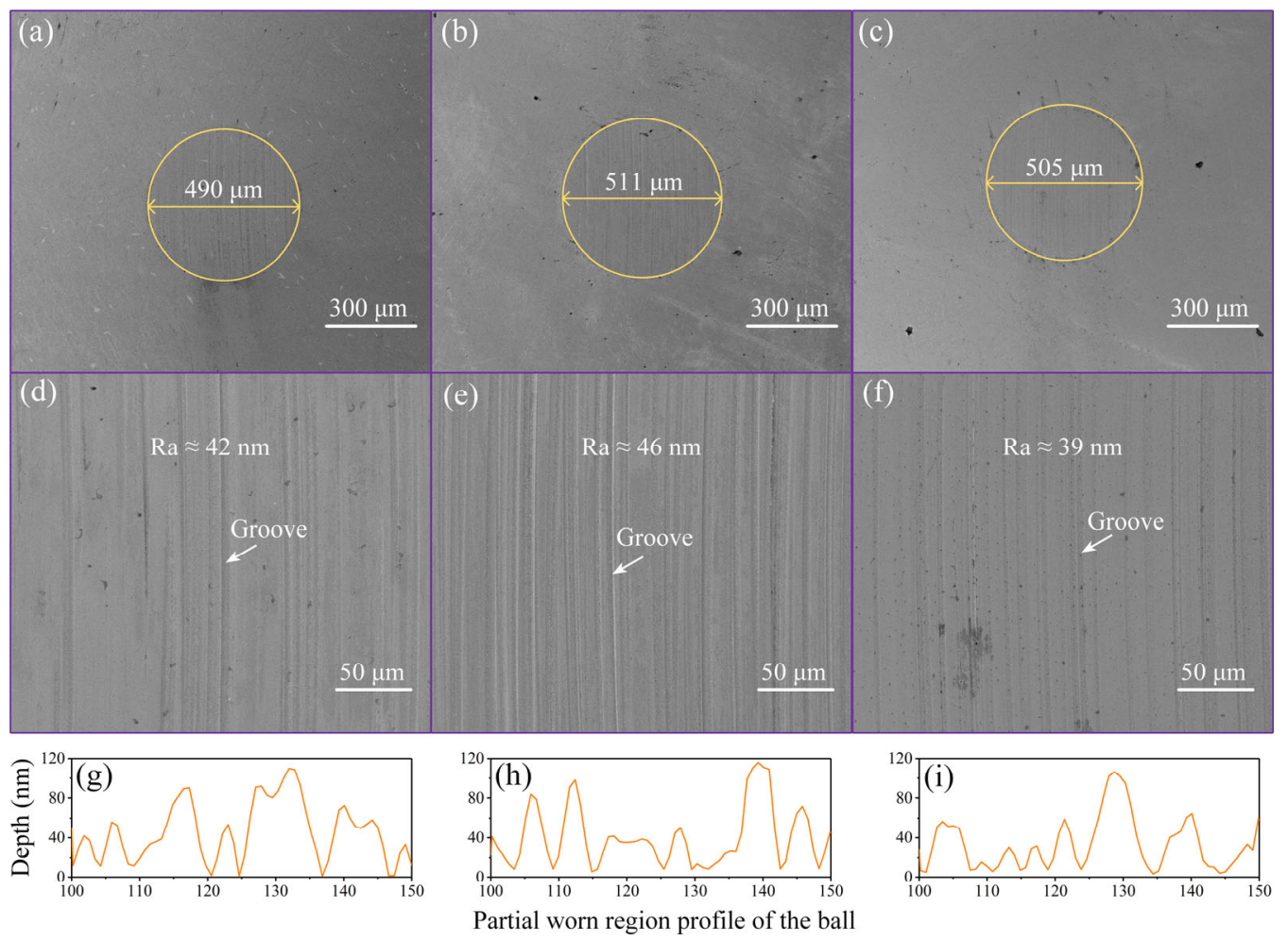

Fig. 3 SEM images of the worn regions on the steel balls that were lubricated with (a, d) PEG(aq), (b, e) PAG100 (the friction pairs were treated by PEG(aq)), and (c, f) PAO6 (the friction pairs were treated by PEG(aq)). (a-c) Overall perspectives and (d-f) detailed images of the worn regions. (g-i) Partial worn region profile of the balls obtained by a 3D white-light interferometry microscope: (g) lubricated with PEG(aq); (h) lubricated with PAG100 (the friction pairs were treated by PEG(aq)); and (i) lubricated with PAO6 (the friction pairs were treated by PEG(aq)). The normal load and sliding velocity were $3 \mathrm{~N}$ and $0.2 \mathrm{~m} / \mathrm{s}$, respectively.

that were treated by PEG(aq), the grooves become more intense and clear, as exhibited in Fig. 3(e). When using PAO6 to lubricate the friction pairs that were treated by PEG(aq), the worn region is slightly smoother than of that lubricated with PEG(aq) and the observed grooves are very shallow, as presented in Fig. 3(f). The worn surfaces of the steel disks are in good agreement with those of the steel balls, and thus, only the latter results are presented. The similar topographies of the worn regions on the steel balls exhibit that the micro-structure on the worn surfaces is formed during the period of treatment with PEG(aq) because of the severe rubbing motion. Tribochemical layers can also form owing to the tribochemical reaction between PEG(aq) and steel. During the superlubricity periods of PAG100 and PAO6, the topography of the steel surfaces remain nearly unchanged, indicating that no severe rubbing occurs between the friction pairs, and thus, there is no tribochemical reaction. During the superlubricity periods, only ordered layers can be formed by PAG100 and PAO6 molecules on the steel surface. Both the tribochemical layers and ordered layers contribute to the robust superlubricity of PAG100 and PAO6.

It is known that PEG and iron can react and form a series of compounds, as presented in Eqs. (1)-(3) [31].

$$
\begin{aligned}
& \mathrm{Fe}+\mathrm{ROH} \rightarrow \mathrm{FeRO}_{\mathrm{ad}}+\mathrm{H}^{+}+\mathrm{e}^{-} \\
& \mathrm{FeRO}_{\mathrm{ad}} \rightarrow \mathrm{FeRO}^{+}+\mathrm{e}^{-} \\
& \mathrm{FeRO}^{+}+\mathrm{H}^{+} \rightarrow \mathrm{Fe}^{2+}+\mathrm{ROH}
\end{aligned}
$$

where $\mathrm{ROH}$ represents the PEG molecule $\left(\mathrm{HO}\left(\mathrm{CH}_{2} \mathrm{CH}_{2} \mathrm{O}\right)_{n} \mathrm{H}\right)$ and $\mathrm{RO}^{-}$denotes its anion $\left(\mathrm{HO}\left(\mathrm{CH}_{2} \mathrm{CH}_{2} \mathrm{O}\right)_{\mathrm{n}}^{-}{ }^{-}\right)$. It is rational to consider that these compounds are generated during the period of treatment with PEG(aq) in our experiment. To explore the chemical characteristics of the worn regions, XPS was performed to explore the chemical elements on the worn regions, as presented in Fig. 4 . The $C 1 \mathrm{~s}$ deconvolution spectrum of PEG(aq) exhibits the 
characteristic peaks of a C-C chain and $\mathrm{C}-\mathrm{H}$ bond at $284.8 \mathrm{eV}$ and a C-O bond at $286.2 \mathrm{eV}$ [32], as presented in Fig. 4(a). The same spectrum applies to PAG100 (where the steel surfaces were treated by PEG(aq) in advance), as shown in Fig. 4(d). The C 1s spectrum of PAO6 (when the steel surfaces are treated by PEG(aq) in advance) is similar to that of PEG(aq) and PAG100, showing a strong peak at $284.8 \mathrm{eV}$ (C-C chain and $\mathrm{C}-\mathrm{H}$ bond) and weak peak at $286.2 \mathrm{eV}$ (C-O bond). This may have resulted from the absence of oxygen atoms in the molecules of PAO6, as displayed in Fig. 4(g). In the O 1s spectra in Figs. 4(b), 4(e), and 4(h), three peaks ranging from 530 to $532 \mathrm{eV}$ are evident. These results indicate that the steel surface and lubricants (PEG(aq), PAG100, and PAO6) can react under a rubbing motion and generate various oxygencontaining complex compounds [33, 34]. In Figs. 4(c), $4(\mathrm{f})$, and $4(\mathrm{i})$, the peaks of Fe $2 \mathrm{p} 3$ ranging from 710 to $714 \mathrm{eV}$ and around $724 \mathrm{eV}$ prove that complex oxygen-containing iron compounds such as iron oxides, $\mathrm{FeOOH}$, and $\mathrm{Fe}(\mathrm{OH})_{3}[35,36]$ are generated and deposited on the steel surfaces. These oxygencontaining iron compounds have proven to be effective boundary lubricants [33-36]. The XPS results demonstrate that on lubrication with PEG(aq) during the treatment period, composite tribochemical layers are formed between each pair of steel surfaces. Moreover, when the lubricant is changed to PAG100 and PAO6, the composite tribochemical layers remain on the surface with little change and contribute to the superlow friction.

In general, film thickness measurements are performed based on the optical interference principle, requiring a steel ball and glass disk with anti-reflection coating on one side and a semi-reflective chromium layer on the other side. Moreover, no wear or wearing-in


Binding energy $(\mathrm{eV})$

Fig. 4 XPS results for the worn regions on the steel ball after being (a-c) lubricated with PEG(aq): (a) C 1s, (b) O 1s, and (c) Fe 2p3; (d-f) lubricated with PAG100 (the friction pairs were treated by PEG(aq)): (d) C 1s, (e) O 1s, and (f) Fe 2p3; and (g-i) lubricated with PAO6 (the friction pairs were treated by PEG(aq)): (g) C 1s, (h) O 1s, and (i) Fe 2p3. The normal load and sliding velocity were $3 \mathrm{~N}$ and $0.1 \mathrm{~m} / \mathrm{s}$, respectively. 
period is allowed during the measurement [37]. In this study, there were wearing-in periods and wear on the steel surfaces, making it inappropriate to measure the film thickness using the optical interference method. Therefore, the film thickness (h) during the superlubricity period was instead estimated using the H-D equation [30]:

$$
h=2.69 \frac{G^{0.53} R U^{0.67}}{W^{0.067}}\left(1-0.61 e^{-0.73 k}\right)
$$

where $G=\alpha E^{\prime}, U=\eta u / E^{\prime} R, W=F / E^{\prime} R^{2}$, and $k \approx 1$ is a coefficient. The pressure-viscosity coefficients $(\alpha)$ of PAG100 and PAO6 are $10 \mathrm{GPa}^{-1}$ and $15 \mathrm{GPa}^{-1}$, respectively $[38,39]$. The viscosity $(\eta)$ is $35 \mathrm{mPa} \cdot \mathrm{s}$ for PAG100 and $47 \mathrm{mPa} \cdot \mathrm{s}$ for PAO6. In this study, the average sliding velocity $(u)$ was $0.075 \mathrm{~m} / \mathrm{s}$ and normal load $(F)$ was $4 \mathrm{~N}$. The equivalent elastic modulus $\left(E^{\prime}\right)$ of a friction pair is given by

$$
E^{\prime}=2 /\left[\left(1-v_{1}^{2}\right) / E_{1}+\left(1-v_{2}^{2}\right) / E_{2}\right]
$$

where $v$ is the Poisson ratio of the material.

To estimate the equivalent radius of the ball $(R)$, the worn region on the ball was regarded as the Hertz elastic deformation region of an equivalent ball under the same normal load $(4 \mathrm{~N})$. Thus, the WSD of the ball $(d=0.49 \mathrm{~mm})$ can be described by Eq. (6) [40]:

$$
\frac{d}{2}=\left(\frac{3 R F}{4 E^{\prime \prime}}\right)^{\frac{1}{3}}
$$

Then, $R$ is given by Eq. (7) [40]:

$$
R=\frac{E^{\prime \prime} d^{3}}{6 F}
$$

where the effective elastic modulus ( $\left.E^{\prime \prime}\right)$ of the two materials can be expressed as

$$
E^{\prime \prime}=1 /\left[\left(1-v_{1}^{2}\right) / E_{1}+\left(1-v_{2}^{2}\right) / E_{2}\right]
$$

Accordingly, $R \approx 570 \mathrm{~mm}$ is obtained, and $h$ between the friction pairs is found to be $211 \mathrm{~nm}$, taking PAG100 as an example. This result indicates the existence of a fluid layer between the steel surfaces.

Based on the above results, a possible mechanism of the superlubricity was proposed, as displayed in
Fig. 5. When the steel surface is treated by PEG(aq), BL can occur between the solid asperity contact region during the initial period (Fig. 5(a)). The asperities rub against each other in the presence of PEG molecules to perform the wearing-in process, which is responsible for the surface smoothening and contact pressure reduction. Moreover, tribochemical layers are formed gradually by the tribochemical reaction between PEG and the steel surfaces (Figs. 5(b) and 5(c)). This composite tribochemical layer consists of iron oxides, $\mathrm{FeOOH}, \mathrm{Fe}(\mathrm{OH})_{3}$, and other oxygen-containing components, which have proven to be effective boundary lubricants [33-36]. When lubricated with PAGs (or PAOs), after the friction pairs are treated by PEG(aq) (Fig. 5(d)), the surface topography is not reformed, as presented in Fig. 3. This indicates that there is no rubbing motion between the asperities, and therefore, no further tribochemical reaction occurs between PAG and the steel surfaces. According to the XPS results, the tribochemical layers formed by the PEG(aq) during the treatment period remain on the steel surfaces.

Even though it is difficult to directly observe the formed ordered layers [9], PAGs [39] and PAOs [8, 11, 41] have been widely used to study TFL owing to their ability to form ordered layers on steel surfaces. Taking PAGs as an example, because they have an ether bond, it makes the molecules highly polar, and thus, they can easily be adsorbed on steel surfaces and form ordered layers. Therefore, it is deduced that in this work, the ordered layers are formed by PAGs and PAOs. Moreover, the velocity- and viscositydependent CoFs presented in Fig. 2 and estimation result of the film thickness suggest that a fluid layer is present between each friction pair. There is high probability of obtaining a full-film EHL owing to the film thickness, in which regime the fluid layer dominates the lubrication condition. In this work, the superlubricity state is attributed to both the tribochemical layers formed during the wearing-in period and the layers formed by PAG and PAO according to the results in Fig. 1. The tribochemical layers are supposed to match with the layers formed by the lubricants (PAG and PAO in this case) to achieve superlubricity. Therefore, all the tribochemical layers, ordered layers, and fluid layer contribute to superlu- 


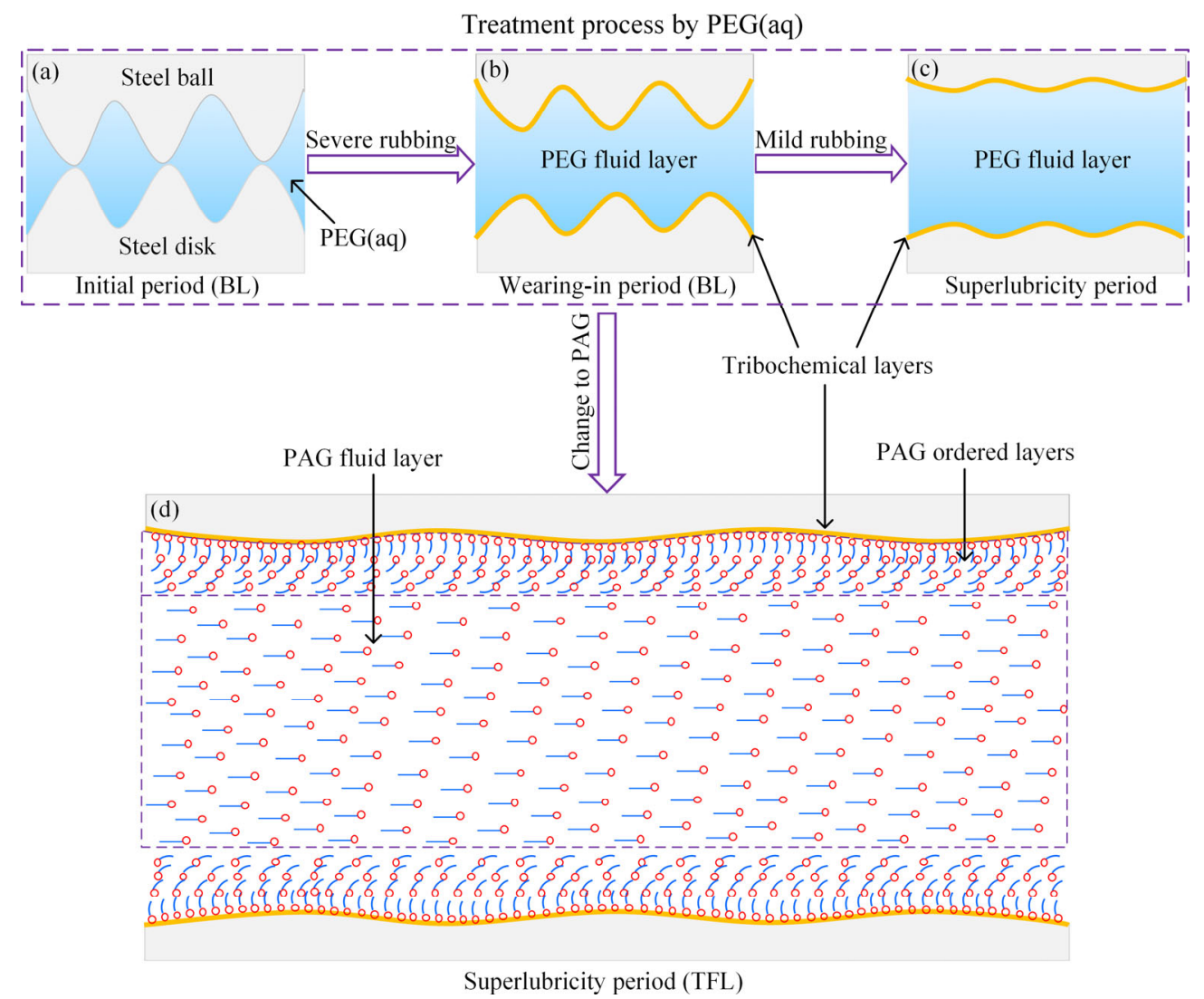

Fig. 5 Proposed superlubricity model in the TFL regime: (a) initial period in the BL regime lubricated with PEG(aq); (b) wearing-in period in the BL regime with PEG(aq) lubrication; (c) superlubricity period with PEG(aq) lubrication; and (d) superlubricity period in the TFL regime with PAG lubrication. (a)-(c) are the treatment processes of the steel/steel friction pairs by PEG(aq).

bricity rather than only the fluid layer, indicating that the lubrication regime is TFL, as presented in Fig. 5(d).

These analyses suggest that the wearing-in period is essential for realizing superlubricity with PAGs or PAOs on steel/steel friction pairs. When a complete wearing-in process with PEG(aq) is performed on the steel/steel friction pairs, superlubricity can be attained when they are lubricated with PAGs and PAOs via the formation of the TFL state between the friction pairs. This work proves that liquid superlubricity can be attained in the TFL regime under particular conditions. Based on the above results, this method of performing a wearing-in process on steel surfaces with PEG(aq) before the lubrication of PAGs and PAOs could be applied in industrial applications. However, a problem is also exposed during this study, i.e., the WSD of the steel balls is extremely large, which is harmful to mechanical components and can reduce the service life of the mechanical equipment. Therefore, we are attempting to improve the superlubricity further based on the proposed superlubricity mechanism in the TFL regime. Moreover, we believe that in the near future, such a new superlubricity system could be designed to lubricate mechanical systems.

\section{Conclusions}

In summary, this work shows that robust superlubricity of PAGs (polar molecules) and PAOs (nonpolar molecules) can be achieved in the TFL regime between steel/steel friction pairs that are treated by PEG(aq) in advance. A plane is formed on the ball by the wearing-in process with PEG(aq), which reduces the contact pressure as well as forms composite tribochemical layers, thereby reducing the friction between the asperities. PAGs and PAOs form ordered layers and fluid layers to protect the tribochemical layer from being sheared off and to reduce the friction between the friction pairs. The attainment of TFL between the steel/steel friction pairs contributes to the robust liquid superlubricity. 
Using the method described in this work, superlubricity can be achieved in the TFL regime by using PAGs and PAOs if the sliding velocity and viscosity can be well matched. The superlubricity attained using PAGs and PAOs is very important for its scientific understanding and will facilitate the design of superlubricity systems on steel surfaces for industrial applications in the future.

\section{Acknowledgements}

This work was financially supported by National Natural Science Foundation of China (Nos. 51775295, 51405256, and 51527901).

Open Access: The articles published in this journal are distributed under the terms of the Creative Commons Attribution 4.0 International License (http:// creativecommons.org/licenses/by/4.0/), which permits unrestricted use, distribution, and reproduction in any medium, provided you give appropriate credit to the original author(s) and the source, provide a link to the Creative Commons license, and indicate if changes were made.

\section{References}

[1] Reynolds O. On the theory of lubrication and its application to $\mathrm{Mr}$. Beauchamp Tower's experiments, including an experimental determination of the viscosity of olive oil. Phil Trans R Soc Lond 177: 157-234 (1886)

[2] Hardy W B, Doubleday I. Boundary lubrication-The paraffin series. Proc R Soc A: Math, Phys Eng Sci 100(707): 550-574 (1922)

[3] Grubin A N, Vinogradova I E. Fundamentals of the hydrodynamic theory of lubrication of heavily loaded cylindrical surfaces. In Investigation of the Contact of Machine Components. Ketova K F, Ed. Moscow: Central Scientific Research Institute for Technology and Mechanical Engineering, 1949.

[4] Lugt P M, Morales-Espejel G E. A review of elastohydrodynamic lubrication theory. Tribol Trans 54(3): 470-496 (2011)

[5] Spikes H A. Sixty years of EHL. Lubr Sci 18(4): 265-291 (2006)

[6] Zhang J, Meng Y G. Boundary lubrication by adsorption film. Friction 3(2): 115-147 (2015)
[7] Luo J B, Wen S Z, Huang P. Thin film lubrication. Part I. Study on the transition between EHL and thin film lubrication using a relative optical interference intensity technique. Wear 194(1-2): 107-115 (1996)

[8] Ma L R, Luo J B. Thin film lubrication in the past 20 years. Friction 4(4): 280-302 (2016)

[9] Gao M, Li H, Ma L, Gao Y, Ma L, Luo J. Molecular behaviors in thin film lubrication, Part two: Direct observation of the molecular orientation near the solid surface. Friction (submitted)

[10] Luo J B, Shen M W, Wen S Z. Tribological properties of nanoliquid film under an external electric field. $J$ Appl Phys 96(11): 6733-6738 (2004)

[11] Liang H, Guo D, Luo J B. Film forming behavior in thin film lubrication at high speeds. Friction 6(2): 156-163 (2018)

[12] Křupka I, Hartl M, Liška M. Thin lubricating films behaviour at very high contact pressure. Tribol Int 39(12): 1726-1731 (2006)

[13] Liang H, Guo D, Luo J B. Experimental investigation of lubrication film starvation of polyalphaolefin oil at high speeds. Tribol Lett 56(3): 491-500 (2014)

[14] Damiens B, Venner C H, Cann P M E, Lubrecht A A. Starved lubrication of elliptical EHD contacts. J Tribol 126(1): 105-111 (2004)

[15] Qiao Y, Zhang S, Liu Y, Ma L, Luo J. Molecular behaviors in thin film lubrication, Part one: Film formation for different polarities of molecules. Friction (submitted)

[16] Li J J, Zhang C H, Deng M M, Luo J B. Investigation of the difference in liquid superlubricity between water- and oil-based lubricants. $R S C A d v$ 5(78): 63827-63833 (2015)

[17] Zeng Q F, Eryilmaz O, Erdemir A. Superlubricity of the DLC films-related friction system at elevated temperature. RSC Adv 5(113): 93147-93154 (2015)

[18] Li J J, Gao T Y, Luo J B. Superlubricity of graphite induced by multiple transferred graphene nanoflakes. Adv Sci 5(3): 1700616 (2018)

[19] Xu J, Li J J. New achievements in superlubricity from international workshop on superlubricity: Fundamental and applications. Friction 3(4): 344-351 (2015)

[20] Zeng Q F, Yu F, Dong G N. Superlubricity behaviors of $\mathrm{Si}_{3} \mathrm{~N}_{4} / \mathrm{DLC}$ films under PAO oil with nano boron nitride additive lubrication. Surf Interface Anal 45(8): 1283-1290 (2013)

[21] Zeng Q F, Dong G N, Martin J M. Green superlubricity of Nitinol 60 alloy against steel in presence of castor oil. Sci Rep 6: 29992 (2016)

[22] Chen Z, Liu Y H, Zhang S H, Luo J B. Controllable superlubricity of glycerol solution via environment humidity. Langmuir 29(38): 11924-11930 (2013) 
[23] Tortora A M, Veeregowda D H. Effects of two sliding motions on the superlubricity and wear of self-mated bearing steel lubricated by aqueous glycerol with and without nanodiamonds. Wear 386-387: 173-178 (2017)

[24] Li H, Wood R J, Rutland M W, Atkin R. An ionic liquid lubricant enables superlubricity to be "switched on" in situ using an electrical potential. Chem Commun 50(33): 43684370 (2014)

[25] Ge X Y, Li J J, Zhang C H, Wang Z N, Luo J B. Superlubricity of 1-ethyl-3-methylimidazolium trifluoromethanesulfonate ionic liquid induced by tribochemical reactions. Langmuir 34(18): 5245-5252 (2018)

[26] Li J J, Zhang C H, Luo J B. Superlubricity behavior with phosphoric acid-water network induced by rubbing. Langmuir 27(15): 9413-9417 (2011)

[27] Ge X Y, Li J J, Zhang C H, Luo J B. Liquid superlubricity of polyethylene glycol aqueous solution achieved with boric acid additive. Langmuir 34(12): 3578-3587 (2018)

[28] Ding M, Lin B, Sui T Y, Wang A Y, Yan S, Yang Q. The excellent anti-wear and friction reduction properties of silica nanoparticles as ceramic water lubrication additives. Ceram Int 44(12): 14901-14906 (2018)

[29] Li J J, Zhang C H, Deng M M, Luo J B. Investigation of the difference in liquid superlubricity between water- and oil-based lubricants. RSC Adv 5(78): 63827-63833 (2015)

[30] Dowson D, Higginson G R. Elasto-Hydrodynamic Lubrication. Oxford (UK): Pergamon Press, 1977.

[31] Guilminot E, Dalard F, Degrigny C. Mechanism of iron corrosion in water-polyethylene glycol (PEG 400) mixtures. Corros Sci 44(10): 2199-2208 (2002)

[32] Soltani T, Lee B K. A benign ultrasonic route to reduced graphene oxide from pristine graphite. J Colloid Interface Sci 486: 337-343 (2017)

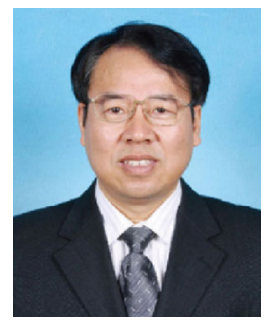

Jianbin LUO. He received his BS degree from Northeastern University in 1982, and got his MS degree from Xi'an University of Architecture and Technology in 1988. In 1994, he received his $\mathrm{PhD}$ degree from Tsinghua University and then joined the faculty of Tsinghua University. Prof. Jianbin Luo is an Academician of the Chinese Academy of Sciences and a Yangtze River Scholar Distinguished
[33] Espinosa T, Sanes J, Bermúdez M D. New alkyletherthiazolium room-temperature ionic liquid lubricants: surface interactions and tribological performance. ACS Appl Mater Interfaces 8(28): 18631-18639 (2016)

[34] Wang H Z, Song Z H, Qiao D, Feng D P, Lu J J. Tribological behavior of $\mathrm{Si}_{3} \mathrm{~N}_{4} / \mathrm{Ti}_{3} \mathrm{SiC}_{2}$ contacts lubricated by lithiumbased ionic liquids. Adv Mater Sci Eng 2014: 863230 (2014)

[35] Song Z H, Liang Y M, Fan M J, Zhou F, Liu W M. Lithium-based ionic liquids as novel lubricant additives for multiply alkylated cyclopentanes (MACs). Friction 1(3): 222-231 (2013)

[36] Amann T, Gatti F, Oberle N, Kailer A, Rühe J. Galvanically induced potentials to enable minimal tribochemical wear of stainless steel lubricated with sodium chloride and ionic liquid aqueous solution. Friction 6(2): 230-242 (2018)

[37] Luo J B, Huang P, Wen S, Li L K Y. Characteristics of liquid lubricant films at the nano-scale. $J$ Tribol 121(4): 872-878 (1999)

[38] Biresaw G, Sharma B K, Bantchev G B, Kurth T L, Doll K M, Erhan S Z, Kunwar B, Scott J W. Elastohydrodynamic properties of biobased heat-bodied oils. Ind Eng Chem Res 53(42): 16183-16195 (2014)

[39] Zhang C H, Zhao Y C, Björling M, Wang Y, Luo J B, Prakash B. EHL properties of polyalkylene glycols and their aqueous solutions. Tribol Lett 45(3): 379-385 (2012)

[40] Wang H D, Liu Y H, Li J J, Luo J B. Investigation of superlubricity achieved by polyalkylene glycol aqueous solutions. Adv Mater Interfaces 3(19): 1600531 (2016)

[41] Xiao H P, Guo D, Liu S H, Lu X C, Luo J B. Experimental investigation of lubrication properties at high contact pressure. Tribol Lett 40(1): 85-97 (2010)

Professor of Tsinghua University, Beijing, China. He was awarded the STLE International Award (2013), the Chinese National Technology Progress Prize (2008), the Chinese National Natural Science Prize (2001), and the Chinese National Invention Prize (1996). Prof. Luo has been engaged in the research of thin film lubrication, superlubricity and tribology in nanomanufacturing. He was invited as a keynote or plenary speaker for more than 20 times on the international conferences. 


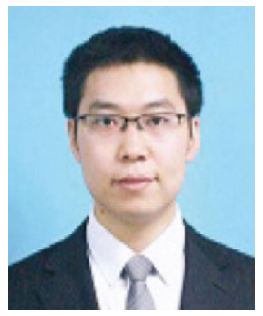

Jinjin LI. He received the BS degree in mechanical engineering from University of Science and Technology of China, Hefei, China, in 2009, and the PhD degree in mechanical engineering from Tsinghua University, Beijing, China in 2014. He is currently an assistant professor at Tsinghua University, Beijing,

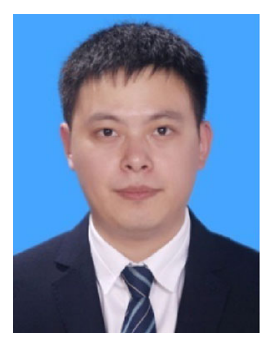

Xiangyu GE. He received the BS degree in mechanical engineering from North China Electric Power University, Beijing, China, in 2010, and the $\mathrm{PhD}$ degree from the same
China. His major research area includes solid and liquid superlubricity, nanotribology and friction theory. He has published more than 30 papers on the international journals (21 papers as the first author). He has been awarded the $4^{\text {th }}$ Hiwin award for outstanding doctoral dissertation, first prize for outstanding doctoral dissertation, and outstanding postdoctor in Tsinghua University.

university in 2016. He is currently a postdoctor at Tsinghua University, Beijng, China. His major research areas include two-dimensional materials lubrication and liquid superlubricity. 